\title{
Progesterone's Serum Level and a New Ultrasonographic Parameter in the First Trimester Pregnancy - Prognostic Factors for Embryonic Demise
}

\author{
Carmen Elena Bucuri ${ }^{1}$ Razvan Ciortea ${ }^{1}$ Andrei Mihai Malutan ${ }^{1}$ Costin Berceanu ${ }^{2}$ \\ Maria Patricia Rada ${ }^{1}$ Dan Mihu ${ }^{1}$
}

${ }^{1}$ Department of Obstetrics and Gynecology, University of Medicine and Pharmacy "Iuliu Hațieganu”, Cluj-Napoca, Romania.

2 Department of Obstetrics and Gynecology, University of Medicine and Pharmacy of Craiova, Craiova, Romania

Address for correspondence Razvan Ciortea, Department of Obstetrics and Gynecology, University of Medicine and Pharmacy "Iuliu Hatieganu”, Cluj-Napoca, 400124, 21st December 1989 Boulevard, no 55-57,Cluj-Napoca, Romania

Rev Bras Ginecol Obstet 2019;41:525-530. (e-mail: r_ciortea@yahoo.com).

\begin{abstract}
\section{Keywords}

- first trimester

- ultrasonography

- progesterone

- prognosis

Objective The etiology of embryonic demise is multifactorial, with chromosomal abnormalities being the most common (40\%). The purpose of the present study is to evaluate the correlation between a serum biomarker, progesterone, and an ultrasonographic parameter, the distance between yolk sac and embryo (DYSE) in assessing the prognosis of pregnancy outcome in the $1^{\text {st }}$ trimester.

Methods The present study is a prospective case-control analysis that includes 2 groups of patients: 81 patients with first-trimester normal evolutive pregnancy and 89 patients with embryonic demise, all of the patients having between 6 and 11 weeks of amenorrhea. Endovaginal ultrasonographic exploration was performed to evaluate the distance between the lower pole of the embryo and the yolk sac. From each subject enrolled in the study, $20 \mathrm{ml}$ of blood was collected for progesterone serum level measurement.

Results Regarding the DYSE in the case group, lower values were observed compared with the control group, the difference being statistically significant. In the statistical analysis of serum progesterone values, statistically significant differences were observed between the 2 groups $(p<0.05)$.

Conclusion The DYSE has a high positive predictive value in identifying pregnancies with potentially reserved outcome, with the present study demonstrating that a DYSE $<3 \mathrm{~mm}$ causes an unfavorable evolution of the pregnancy. Low serum levels of progesterone are associated with an increased rate of nonviable embryos. The correlation between these two parameters increases the effectiveness of screening methods in prenatal monitoring and improves the diagnostic methods for the firsttrimester pregnancies whose outcome potential can be reserved.
\end{abstract}

(D) Carmen Elena Bucuri's ORCID is https://orcid.org/0000-00023422-6847.

received

November 20, 2018

accepted

July 15, 2019
DOI https://doi.org/

10.1055/s-0039-1696948. ISSN 0100-7203.
Copyright $\odot 2019$ by Thieme Revinter

Publicações Ltda, Rio de Janeiro, Brazil
License terms

(c) (i) 


\section{Introduction}

One in four pregnant women will miscarry at some time during her reproductive lifetime. The incidence of early embryonic demise is higher compared with other early pregnancy complications. ${ }^{1}$ In $>40 \%$ of the cases, the etiology of embryonic demise are chromosomal abnormalities. ${ }^{2}$ Successful blastocyst implantation requires precise synchronization between the embryo, the uterine, and the hormonal environment. ${ }^{3}$ Ultrasonographic examination is the method of choice in the diagnosis of embryonic demise. ${ }^{4}$ In addition to this technique, another sensitive and specific biomarker is required to determine the viability of the early pregnancy. ${ }^{5}$ This pathology determines a standardized management that is associated with an increased emotional impact. ${ }^{6}$

Progesterone is a 21-carbon steroid hormone secreted by the corpus luteum of the ovary. This hormone is an important promoting factor for endometrial decidualization, preparing the uterus for implantation of the blastocyst, and for maintaining the pregnancy. The physiological functions of progesterone include inhibition of smooth muscle contractility and inhibition of immune responses like those involved in graft rejection. ${ }^{7}$ Obviously, it is essential to study women after natural conceptions without exogenous progesterone support, while evaluating the relation between serum progesterone and viability of the first-trimester pregnancy. 8,9 There are studies that suggest that serum progesterone measured in early pregnancy is the most powerful single predictor of pregnancy outcome in natural conceptions. ${ }^{10}$

Ultrasound evaluation is the method of choice for assessing outcome prognosis of the early pregnancy. One in four women at some point in her reproductive lifetime suffers an abortion. ${ }^{11}$ There are no prospective data to outline the guidelines for ultrasound diagnosis as accurately as possible for embryonic demise. The results are limited by the small number of studies and patients, by studies conducted long ago, and by the variable reference standards for the diagnosis of early embryonic death. However, the endovaginal ultrasonography technique has become a valuable tool in the solid assessment of pregnancy and has helped to establish new elements about its evolution. The ultrasonography diagnosis of the first-trimester pregnancy with an unfavorable outcome was based on a variety of elements, represented by absence of the yolk sac (YS), lack of identification of the embryo or of its cardiac activity, and abnormalities of the gestational sac (GS). ${ }^{12}$

The identification of other prognosis ultrasonographic elements has also been required. In the early embryonic development, the embryo is immediately revealed adjacent to the YS, and the embryonic structure called yolk stalk has not developed yet. The separation of the embryo from the YS is due to the development both of this structure and of the embryo. Thus, the distance between the embryo and the YS is increased due to the growth of the yolk stalk. For embryos with crown-rump length (CRL) $\leq 5 \mathrm{~mm}$, there must be no separation of the YS. ${ }^{13}$ When the embryo reaches $5 \mathrm{~mm}$, there is a physiological separation between the embryo and the YS due to the development of this structure. The absence of separation of the embryo from the YS, when the CRL exceeds $5 \mathrm{~mm}$ and the embryo is with cardiac activity, is considered an unfavorable prognosis factor. ${ }^{14}$ Filly et al ${ }^{12}$ argue that this ultrasonographic parameter is valuable in anticipating an unfavorable outcome of the pregnancy, wether used alone or in combination with another prognostic ultrasonographic parameter. ${ }^{15}$

The aim of the present study is to evaluate the correlation between a serum biomarker (progesterone) and an ultrasonographic parameter, the distance between yolk sac and embryo (DYSE), in assessing the prognosis of pregnancy outcome in the $1^{\text {st }}$ trimester.

\section{Methods}

The present study is a case-control prospective analysis that includes 170 patients in the $1^{\text {st }}$ trimester of pregnancy without associated pathology. The patients were followedup for a period of 2 years, between 2016 and 2017. The patients were divided into 2 groups: the control group of 81 first-trimester pregnancy patients, and the case group of 95 pregnant patients with a potentially reserved outcome pregnancy with between 6 and 11 weeks of amenorrhea. All of the 95 patients showed unfavorable prognosis ultrasonographic signs, that is, a DYSE $<3 \mathrm{~mm}$ at a gestational age in which the separation would have already occurred; however for 6 of these patients, the prediction of the ultrasonographic parameters studied of an unfavorable evolution was not confirmed by repeated examinations. Thus, the case group included 89 patients with embryonic demise.

Endovaginal ultrasound with a $6.5 \mathrm{MHz}$ nominal frequency Toshiba Aplio 300 probe (Toshiba, Tokyo, Japan) was performed in dorsal decubitus with bent knees in order to identify the predicted outcome prognosis factors: CRL, GS size, appearance and size of the YS and the distance between the lower embryo pole and the DYSE. There were on average between 2 and 3 serial examinations, performed at regular intervals of between 3 and 5 days, also by the same examiner, until the final diagnosis of embryonic demise. The measurements of DYSE were performed using the sagittal section and evaluating three different distances from the inferior pole of the embryo to the YS, the smallest from those 3 distances being taken into account (-Figs. 1 and $\mathbf{2}$ ).

From each subject enrolled in the present study, $20 \mathrm{ml}$ of blood was collected by venipuncture into anticoagulant-free tubes for progesterone serum level measurements. The centrifuge serum was divided and stored in $600 \mu$ freezing tubes at $-60^{\circ} \mathrm{C}$ until the specimens were processed to avoid repeated freeze-thaw cycles.

The privacy and dignity of the participants have been respected. In this respect, the participation of the patients in the research activities was made only after obtaining their informed consent. Also, the working methodology has been obtained by the Ethics Commission of the University of Medicine and Pharmacy "Iuliu Hatieganu”, Cluj-Napoca, Romania.

\section{Statistical Analysis}

The descriptive statistics elements were calculated, and the data was presented using centrality, location and 


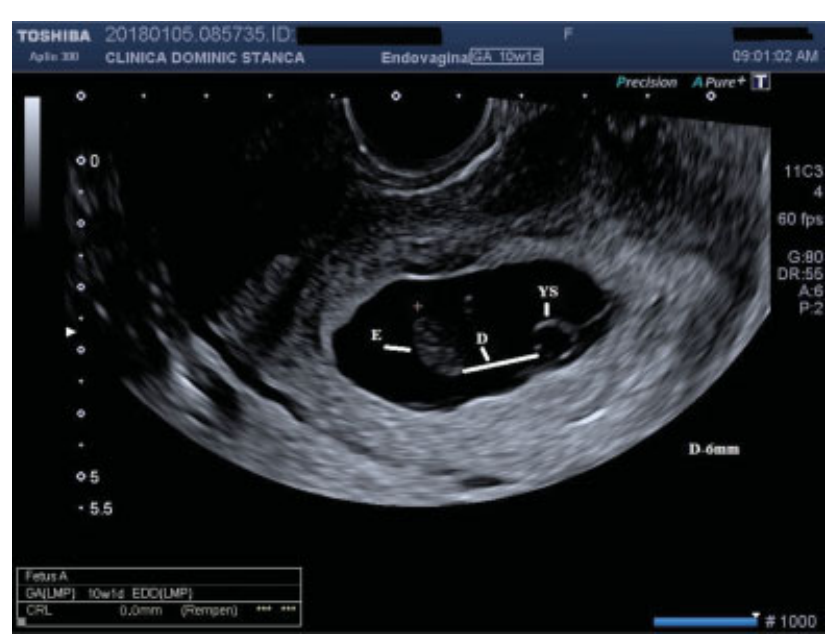

Fig. 1 The distance between yolk sac and embryo in a physiological first-trimester pregnancy.

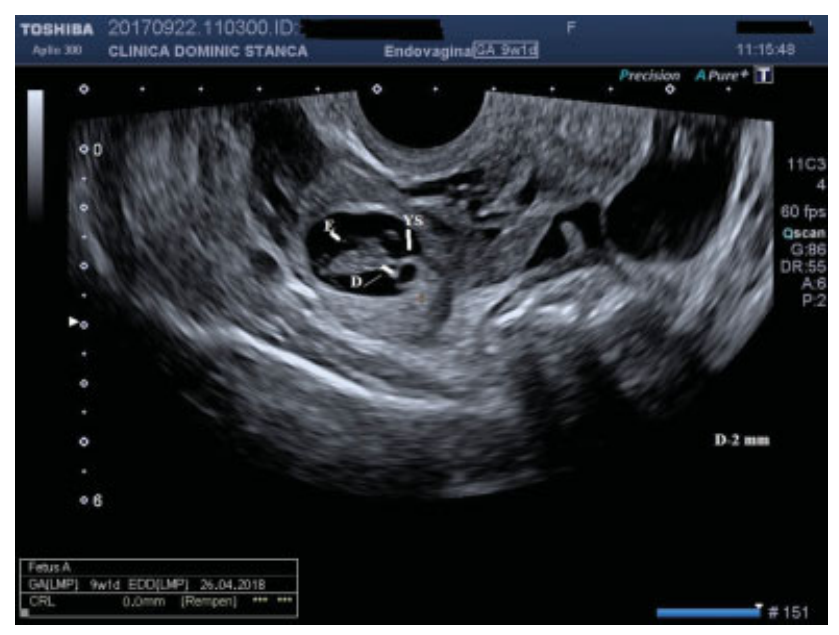

Fig. 2 The distance between yolk sac and embryo in a pregnancy with an embryonic demise.

distribution indicators. The Shapiro-Wilk test was used to test normal distribution. The variance was tested with the $\mathrm{F}$ test. In the case of normal distribution of data, the Student ttest was used, and in the case of nonuniform distribution values or ranks, the nonparametric Mann-Whitney U test was used for two nonpaired samples. For the analysis of three or more samples, the analysis of variance (ANOVA) test was used for normal distribution data, or the Kruskal-Wallis nonparametric test for nonuniform values or ranges. The significance threshold for the tests used was $\alpha=0.05$ (5\%), 0.01 ( $1 \%$ ) or 0.001 . The Pearson correlation coefficient ( $r$ ) was used to detect the correlation between two continuous quantitative variables with normal (uniform) distribution. In the case of nonuniform variables, the Spearman correlation coefficient $(\rho)$ was used. The analysis of correlation coefficients was performed using the Colton rule. Polynomial regression was used to obtain the mathematical equation of the dependence of a variable on another variable.

\section{Results}

No statistically significant difference between the 2 groups $(p>0.05)$ was observed in the statistical analysis of the gestational age (GA) (-Table 1).

No statistically significant difference between the two groups ( $p>0.05$ ) was observed in the statistical analysis of the CRL values. Of the 170 patients, 81 patients (47.65\%) presented embryos with cardiac activity and with the CRL ranging between $6.7 \mathrm{~mm}$ and $44.1 \mathrm{~mm}$, and 89 patients (52.35\%) presented embryos with no cardiac activity with the CRL ranging between $6.6 \mathrm{~mm}$ and $33.4 \mathrm{~mm}$ (-Table 2 ).

In the statistical analysis of DYSE values, statistically significant differences were observed between the 2 groups $(p<0.001)$ in all of the weeks of amenorrhea studied ( - Figs 3 and 4). Thus, it was shown that a DYSE of $<3 \mathrm{~mm}$ correlated with an unfavorable prognosis of pregnancy evolution (-Tables 3 and 4 ).

\section{Discussion}

Recent studies suggest that serum progesterone measured in early pregnancy is the most powerful single predictor of pregnancy outcome in natural conceptions. ${ }^{1}$ Progesterone, regarded as the pregnancy hormone, coordinates a series of complex events that ultimately lead to the synchronized

Table 1 Comparative analysis and statistical significance for gestational age values in the studied groups

\begin{tabular}{|l|l|l|l|l|l|l|l|l|}
\hline Indicators & Group & Average & Error Standard & Median & Standard Deviation & Min & Max & $p$-value \\
\hline \multirow{2}{*}{ Gestational age } & $\mathrm{I}$ & 8.49 & 0.1767 & 9 & 1.5900 & 6 & 11 & 0.779 \\
\cline { 2 - 9 } & $\mathrm{II}$ & 8.56 & 0.1721 & 9 & 1.6234 & 6 & 11 & \\
\hline
\end{tabular}

Table 2 Comparative analysis and statistical significance of the values of the ultrasonographic indicators in the studied groups

\begin{tabular}{|l|l|l|l|l|l|l|l|l|}
\hline Indicators & Group & Average & Error Standard & Median & Standard Deviation & Min & Max & $p$-value \\
\hline $\begin{array}{l}\text { Distance between } \\
\text { yolk sac and embryo }\end{array}$ & I & $5.57 \mathrm{~mm}$ & 0.1480 & 5 & 1.3317 & 3 & 9 & $<0.0001$ \\
\hline & $\mathrm{II}$ & $2.63 \mathrm{~mm}$ & 0.1511 & 2.3 & 1.4251 & 1 & 8 & \\
\hline Crown-rump length & $\mathrm{I}$ & $20.8 \mathrm{~mm}$ & 0.1224 & 22 & 1.1019 & 6 & 43 & 0.49 \\
\hline & $\mathrm{II}$ & $19.5 \mathrm{~mm}$ & 0.1068 & 18 & 1.0080 & 6 & 42 & \\
\hline
\end{tabular}




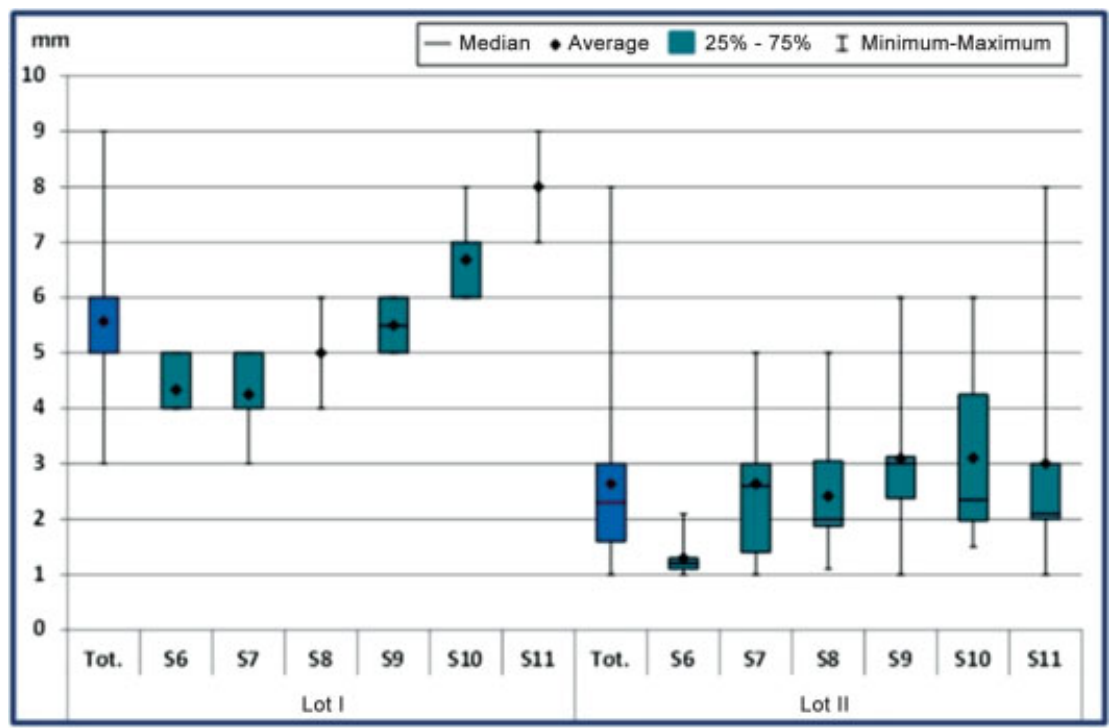

Fig. 3 The distance between yolk sac and embryo in the studied groups and subgroups.

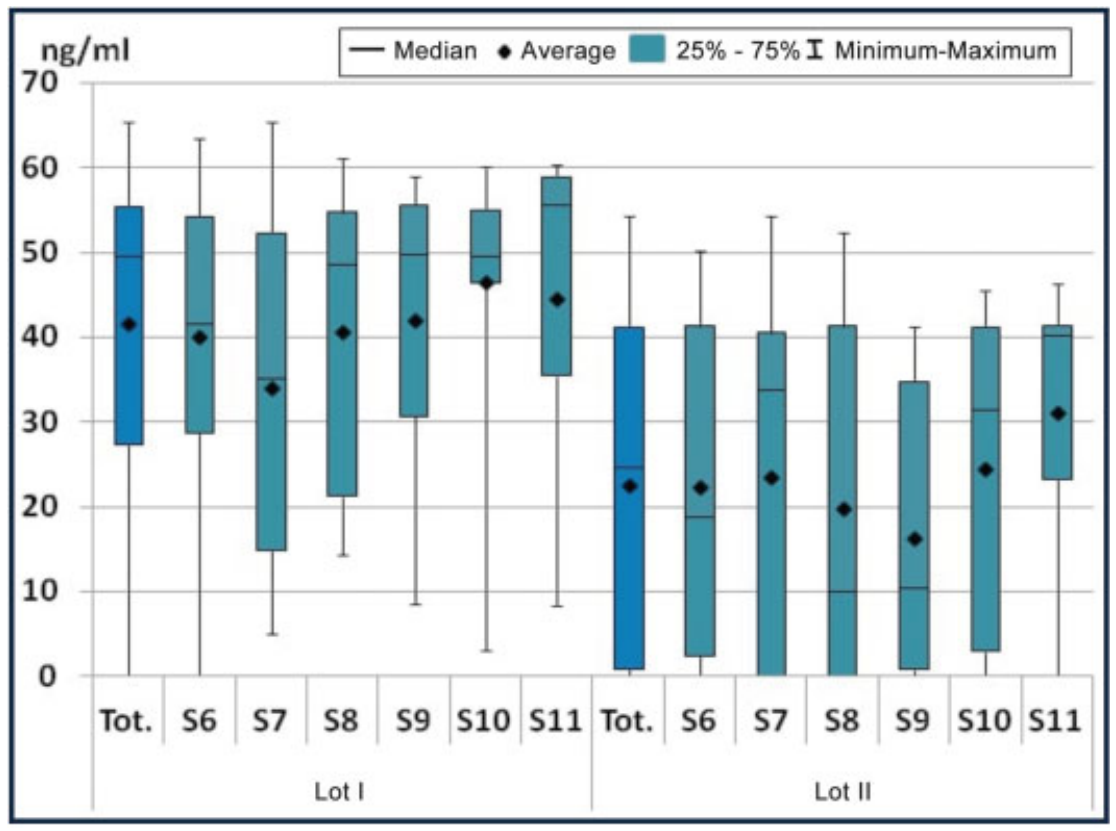

Fig. 4 Comparative analysis for progesterone values in the studied subgroups.

Table 3 Comparative analysis and statistical significance of the values of progesterone in the studied groups

\begin{tabular}{|l|l|l|l|l|l|l|l|l|}
\hline Indicator & Lot & Average & Error Standard & Median & Standard Deviation & Min & Max & $p$-value \\
\hline Progesterone & I & 41.62 & 1.9984 & 49.61 & 17.9853 & 0.14 & 65.42 & $<0.0001$ \\
\cline { 2 - 8 } & II & 22.58 & 2.0513 & 24.758 & 19.3516 & 0.00531 & 54.31 & \\
\hline
\end{tabular}

Table 4 Statistical analysis of the correlation between the values of the indicators studied

\begin{tabular}{|l|l|l|l|l|l|}
\hline \multicolumn{2}{|l|}{ Indicator } & \multicolumn{1}{l|}{ Lot I } & \multicolumn{2}{l|}{ Lot II } \\
\hline DYSE & $\mathrm{P}$ & 0.1919 & ${ }^{* *}$ & 0.0977 & $* *$ \\
\hline
\end{tabular}

Abbreviations: DYSE, the distance between yolk sac and embryo;

P, progesterone.

**a good correlation between the indicators studied development of the embryo and the differentiation of uterine cells for implantation. Decreased progesterone levels lead to abortion. ${ }^{16}$ Therefore, the present prospective study was designed to detect the relation between serum progesterone and the viability of the pregnancy during the $1^{\text {st }}$ trimester. Our data suggest the possibility that the early stages of pregnancy may be particularly sensitive to progesterone deficiency. If the decrease of systemic progesterone is one 
of the main mechanisms by which inflammation induces pregnancy loss, our results reinforce the benefit of using progesterone to reduce the risk of miscarriage. Based on these findings, we suggest that the functional network between hormones, cytokines and hormonal mediators at the fetomaternal interface has a fundamental role in the development of a successful pregnancy. A defect in the integrity of this network probably leads to pregnancy loss.

Daily et $\mathrm{al}^{17}$ found that the mean serum progesterone was significantly higher for viable pregnancies $(22.1 \mathrm{ng} / \mathrm{mL}$ ) compared with nonviable pregnancies (10.1 ng/ mL), and they concluded that a serum progesterone assay alone is predictive of pregnancy outcome, especially during the first 8 weeks of gestation. Taghavi ${ }^{18}$ found that the serum progesterone levels were significantly higher in patients with viable pregnancies $(20.48 \pm 6.066 \mathrm{ng} / \mathrm{mL})$ compared with patients with nonviable pregnancies ended by spontaneous abortion $(7.78 \pm 2.06 \mathrm{ng} / \mathrm{mL})$; this author concluded that serum progesterone alone is a reliable marker for the prediction of early pregnancy failure. ${ }^{18}$

In our study, $6.7 \%$ of the viable pregnancies had serum progesterone levels $<10 \mathrm{ng} / \mathrm{mL}$, while $20.7 \%$ of the nonviable pregnancies had serum progesterone levels $>10 \mathrm{ng} / \mathrm{mL}$; the serum progesterone at a cutoff level of $10 \mathrm{ng} / \mathrm{mL}$ was $79.3 \%$ sensitive for the diagnosis of nonviable pregnancy, and was 93.3\% specific for diagnosing viable pregnancies. Also in the present study, $1.1 \%$ of the viable pregnancies had a serum progesterone level $<20 \mathrm{ng} / \mathrm{mL}$, while $4.8 \%$ of the nonviable pregnancies had a serum progesterone level $>20 \mathrm{ng} / \mathrm{mL}$; serum progesterone at a cutoff level of $20 \mathrm{ng} / \mathrm{mL}$ was 95.1\% sensitive for diagnosing nonviable pregnancies, and was $98.9 \%$ specific for the diagnosis of viable pregnancies.

Al-Sebai et al $^{19}$ concluded that a single serum progesterone measurement taken in early pregnancy is valuable in the immediate diagnosis of early pregnancy failure and in the long-term prognosis of viability. Also, the result of the present study suggests that serum progesterone is a reliable marker for early pregnancy failure, and that a single assay of its serum level can differentiate between viable and nonviable pregnancies. Future trials and large population studies are needed to support our findings and to establish the cutoff values of serum progesterone to differentiate between viable and nonviable pregnancies.

The markers that emerged from the present study as predictors of viability have known and established roles in the assessment of healthy pregnancies. As a product of the corpus luteum in early pregnancy, and later the placenta, progesterone has been extensively evaluated as a predictor of early pregnancy failure. A meta-analysis of 26 studies suggested that serum progesterone $<5 \mathrm{ng} / \mathrm{mL}$ had good prediction for nonviable pregnancies. ${ }^{20}$

Laing et $\mathrm{al}^{15}$ consider that identifying a DYSE of $<3 \mathrm{~mm}$ in size, evidenced in pregnancies with $\mathrm{CRL}>5 \mathrm{~mm}$ and an embryo without cardiac activity, is defining for the diagnosis of embryonic demise. ${ }^{15,21}$ In the absence of the specific ultrasonographic signs of an unfavorable prognosis of the outcome of first-trimester pregnancies, such as hypotonic GS, YS of increased size, embryonic bradycardia, identifying a
DYSE $<3 \mathrm{~mm}$ at an early stage of pregnancy, could increase the accuracy of the diagnosis. ${ }^{12}$

Prospective data on which precise prognosis factors can be established to determine a series of decisive predictive factors in the ultrasonographic diagnosis of first-trimester pregnancies with a potentially reserved outcome are insufficient. Transvaginal ultrasound has become an important tool for assessing first-trimester pregnancies and has helped to identify new parameters of pregnancy outcome.

The data from the present study shows that the DYSE is an ultrasonographic feature that indicates earlier embryonic demise compared with specific ultrasonographic parameters, which certainly confirms the absence of pregnancy evolution at a more advanced GA. For higher diagnostic accuracy, it is important to correlate this parameter with a serum marker. The clinical value of the results of the present study consists in the possibility of using these markers in establishing a management since the early stages of pregnancy, in the case of patients who have ultrasonographic parameters considered to have an unfavorable prognosis. Most previous studies that have shown the ability of serum biomarkers to anticipate embryonic demise have been performed on cohorts of patients already experiencing symptoms of spontaneous abortion (bleeding and abdominal pain). ${ }^{22}$ By this association, the originality of the present study is outlined: the correlation of this ultrasound parameter with an important hormone, which is highly used in the literature in the prediction of some pathological entities that appear late in pregnancy, but which is known to have an important role in the $1 \mathrm{st}$ trimester of pregancy.

\section{Conclusion}

The DYSE has a high positive predictive value in identifying potentially reserved outcome of pregnancies, demonstrating in the present study that a DYSE $<3 \mathrm{~mm}$ leads to an unfavorable evolution of the pregnancy. Also, correlating this ultrasound parameter with a serologic one, that is, with the progesterone serum level, brings valuable information about the viability of the pregnancy in the $1^{\text {st }}$ trimester. Low progesterone serum levels of are associated with an increased rate of nonviable embryos.

\section{Contributors}

All of the authors contributed with the project and data interpretation, the writing of the article, the critical review of the intellectual content, and with the final approval of the version to be published.

Conflicts of Interests

The authors have no conflicts of interests to declare.

\section{References}

1 Elson J, Salim R, Tailor A, Banerjee S, Zosmer N, Jurkovic D. Prediction of early pregnancy viability in the absence of an ultrasonically detectable embryo. Ultrasound Obstet Gynecol 2003;21(01):57-61. Doi: 10.1002/uog.1 
2 Azmanov DN, Milachich TV, Zaharieva BM, et al. Profile of chromosomal aberrations in different gestational age spontaneous abortions detected by comparative genomic hybridization. Eur J Obstet Gynecol Reprod Biol 2007;131(02):127-131. Doi: 10.1016/j.ejogrb.2006.04.037

3 Jauniaux E, Johns J, Burton GJ. The role of ultrasound imaging in diagnosing and investigating early pregnancy failure. Ultrasound Obstet Gynecol 2005;25(06):613-624. Doi: 10.1002/uog.1892

4 Woods-Giscombé CL, Lobel M, Crandell JL. The impact of miscarriage and parity on patterns of maternal distress in pregnancy. Res Nurs Health 2010;33(04):316-328. Doi: 10.1002/nur.20389

5 Bourdiec A, Shao R, Rao CV, Akoum A. Human chorionic gonadotropin triggers angiogenesis via the modulation of endometrial stromal cell responsiveness to interleukin 1: a new possible mechanism underlying embryo implantation. Biol Reprod 2012; 87(03):66. Doi: 10.1095/biolreprod.112.100370

6 Hanita $\mathrm{O}$, Hanisah AH. Potential use of single measurement of serum progesterone in detecting early pregnancy failure. Malays J Pathol 2012;34(01):41-46

7 Abdelazim IA, Elezz AA, Elsherbiny M. Relation between single serum progesterone assay and viability of the first trimester pregnancy. Springerplus 2012;1(01):80. Doi: 10.1186/2193-1801-1-80

8 Vicdan K, Zeki Isik A. Luteal phase hormonal profile in prediction of pregnancy outcome after assisted reproduction. Eur J Obstet Gynecol Reprod Biol 2001;96(01):98-101. Doi: 10.1016/S0301-2115 (00)00400-0

9 Al Jufairi ZAA. The value of serum progesterone measurement in early pregnancy. Bahrain Med Bull 2000;22(01):1-6

10 Phipps MG, Hogan JW, Peipert JF, Lambert-Messerlian GM, CanickJA, Seifer DB. Progesterone, inhibin, and hCG multiple marker strategy to differentiate viable from nonviable pregnancies. Obstet Gynecol 2000;95(02):227-231. Doi: 10.1016/S0029-7844(99)00480-9

11 Royal College of Obstetricians and Gynaecologists. Management of Early Pregnancy Loss. London: RCOG Press; 2006

12 Filly MR, Callen PW, Yegul NT, Filly RA. The yolk stalk sign: evidence of death in small embryos without heartbeats. JUltrasound Med 2010;29(02):237-241. Doi: 10.7863/jum.2010.29.2.237

13 Aziz S, Cho RC, Baker DB, Chhor C, Filly RA. Five-millimeter and smaller embryos without embryonic cardiac activity: outcomes in women with vaginal bleeding. JUltrasound Med 2008;27(11): 1559-1561. Doi: 10.7863/jum.2008.27.11.1559
14 Rowling SE, Langer JE, Coleman BG, Nisenbaum HL, Horii SC, Arger PH. Sonography during early pregnancy: dependence of threshold and discriminatory values on transvaginal transducer frequency. AJR Am J Roentgenol 1999;172(04):983-988. Doi: 10.2214/ajr.172.4.10587132

15 Laing FC, Frates MC, Benson CB. Ultrasound evaluation during the first trimester of pregnancy. In: Callen PW, ed. Ultrasonography in Obstetrics and Gynecology. 5th ed. Philadelphia, PA: Saunders Elsevier; 2008:181-224

16 Aisemberg J, Vercelli CA, Bariani MV, Billi SC, Wolfson ML, Franchi AM. Progesterone is essential for protecting against LPS-induced pregnancy loss. LIF as a potential mediator of the anti-inflammatory effect of progesterone. PLoS One 2013;8(02):e56161. Doi: 10.1371/journal.pone.0056161

17 Daily CA, Laurent SL, Nunley WC Jr. The prognostic value of serum progesterone and quantitative beta-human chorionic gonadotropin in early human pregnancy. Am J Obstet Gynecol 1994;171 (02):380-383, discussion 383-384. Doi: 10.1016/s0002-9378 (94)70038-9

18 Taghavi AH. Detection of changes of hCG, progesterone and estradiol serum levels in threatened abortion in the first three months of gestation. Majallah-i Ghudad-i Darun/Riz va Mitabulism-i Iran 2004;6:163-169

19 al-Sebai MA, Kingsland CR, Diver M, Hipkin L, McFadyen IR. The role of a single progesterone measurement in the diagnosis of early pregnancy failure and the prognosis of fetal viability. $\mathrm{Br} \mathrm{J}$ Obstet Gynaecol 1995;102(05):364-369. Doi: 10.1111/j.1471-0528.1995. tb11286.x

20 Mol BW, Lijmer JG, Ankum WM, van der Veen F, Bossuyt PM. The accuracy of single serum progesterone measurement in the diagnosis of ectopic pregnancy: a meta-analysis. Hum Reprod 1998;13(11):3220-3227. Doi: 10.1093/humrep/13.11.3220

21 Salamanca A, Fernández-Salmerón P, Beltrán E, Mendoza N, Florido J, Mozas J. Early embryonic morphology sonographically assessed and its correlation with yolk sac in missed abortion. Arch Gynecol Obstet 2013;287(01):139-142. Doi: 10.1007/ s00404-012-2499-8

22 Duan L, Yan D, Zeng W, Yang X, Wei Q. Predictive power progesterone combined with beta human chorionic gonadotropin measurements in the outcome of threatened miscarriage. Arch Gynecol Obstet 2011;283(03):431-435. Doi: 10.1007/s00404-010-1367-7 